\title{
Analysis of the Effect of Health Education Activities on Selected Child Health Determinants in Two Communities of Ile Ife, Nigeria
}

\author{
Olorunfemi Akinbode Ogundele ${ }^{1 *}$, Tolulope Ogundele ${ }^{2}$
}

\footnotetext{
OPEN ACCESS

Citation: Olorunfemi Akinbode Ogundele, Tolulope Ogundele. Analysis of the Effect of Health Education Activities on Selected Child's Health Determinants in Two Communities of Ile Ife, Nigeria. Ethiop J HealthSci.2018;28(2):217.

doi:http://dx.doi.org/10.4314/ejhs.v28i2.13 Received: November 1, 2017

Accepted: November 3, 2017

Published: March 1, 2018

Copyright: (C) 2018 Birkneh Taddese., et al. This is an open access article distributed under the terms of the Creative Commons Attribution License, which permits unrestricted use, distribution, and reproduction in any medium, provided the original author and source are credited.

Funding: Nil

Competing Interests: The authors declare that this manuscript was approved by all authors in its form and that no competing interest exists.

Affiliation and Correspondence:

${ }^{1}$ Department of Community Medicine and Primary Care, State Specialist Hospital, Ondo City, Ondo State, Nigeria

${ }^{2}$ Department of Paediatrics and Child Health, State Specialist Hospital, Ondo City, Ondo State, Nigeria

*email:femidele@gmail.com
}

\begin{abstract}
BACKGROUND: Child survival in Nigeria is threatened not just by the direct causes of childhood mortality such as the common childhood diseases but also by the associated child health determinants. Health education activities as part of community integrated management of childhood illness have the potential to improve these child health determinants. The objective of this study was to assess the effect of community level health education activities on selected child health determinants in Ile Ife, Nigeria. METHODS. A cross-sectional comparative study was carried out among 722 caregivers in two LGAs of Osun State, Nigeria, using a multistage cluster sampling method. Data were collected from mothers of index children aged 0-59 months using an intervieweradministered questionnaire.
\end{abstract}

RESULTS. Vitamin A supplementation before the study was received by $89.1 \%$ and $79.8 \%$ of the children aged 9 months old and above in implementing and non-implementing LGAs (p=0.002). Amongst children age 12-23 months in the implementing LGA, 93.4\% received DPT3, (91.8\%) received measles compared with non-implementing LGA in which $66.7 \%$ received DPT and (64\%) measles. Children in the CIMCI implementing $L G A$ were three times more likely to receive DPT Vaccine (Adjusted odds ratio (AOR) 3.02, 95\% confidence interval (CI) 1.55-7.98), and five and half times more likely to receive measles vaccine (AOR 5.56, 95\% CI 2.76-12.54).

CONCLUSION. The study concluded that community level health education activities have a positive effect on child health practices and determinants.

KEYWORDS: Community, Immunisation, CIMCI Child health, Health education, Mothers, Determinants

\section{INTRODUCTION}

The last three decades witnessed a lot of activities aimed at achieving improvement in child survival. Child health programmes were targeted not just at direct causes of childhood mortality but also at selected child health determinants known to contribute to these deaths. These efforts were directed at key practices such as immunisation and vitamin supplementation, hand washing practices,

DOI: http://dx.doi.org/10.4314/ejhs.v28i2.13 
faecal disposal, etc, all in an attempt to decrease under-five mortality. According to the United Nations mortality estimates, Nigeria achieved only an average of $1.2 \%$ reduction in under-five mortality per year since 1990; and needed to have achieved an annual reduction rate, of $10 \%$ to have met MDG 4 goal in 2015 (1). With the move to Sustainable Development Goals (SDGs) internationally, child health remains a key area of focus in the SDGs that relate to health. Success in SDG goal 3.2 (ending preventable child deaths) will depend on how well the country will progress and sustain other broad range of health determinants which have been operational and successful in the country.

Child health determinants activities such as immunisation and Vitamin A supplementation, sanitation practices and clean water supply are core activities in the Community integrated management of Childhood illness (CIMCI) programme which operates in Ife Central Local Government Area, of Osun State, Nigeria. Community Resource Persons (CORPs) were trained and equipped with skills for this purpose and other functions at the beginning of the programme. It has been documented that child mortality could be reduced by over $23 \%$ by improving the intake of Vitamin A through diet or supplementation in areas with Vitamin A deficiency (2). Most of the deaths from vaccine preventable diseases each year can be prevented if all children received immunization before the age of one year. In spite of diarrhoea deaths falling from 1.2 million to 526,000 in 2015 , diarrhoea illness still kills more than 1,400 children underfive every day (3). Improved hygiene practices, including safe disposal of excreta and hand washing, can reduce the incidence of diarrhoea by more than $10 \%$ (4). To provide care for children, families need knowledge, skills, motivation and support from the community. The aim of CIMCI is to initiate, reinforce and sustain family practices and skills that are important in reducing childhood mortality (5). Health education activities were central amongst other functions of CORPs trained for this programme. These CORPs educated mothers and mobilized them for immunization.
CORPs also trained, educated and encouraged caregivers on proper hand washing, safe water and faecal disposal practices. They regularly visited these caregivers in order to reiterate, motivate and sustain these practices (6). It is of immense benefit to assess the effect of this sustained health education by CORPs on selected child health determinants after six years of CIMCI activities, as findings from the assessment can be of help for policy makers and for program action. This study was aimed at assessing the effect of community level health education activities on selected Child health determinants in Ile Ife, Nigeria.

\section{METHODS}

Study sites and period: The study was conducted in two LGAs of Osun State in South-West Nigeria. The study was done between April and June in 2013 as the second phase of an initial study 6 months earlier between October and December, 2012. The purpose of the initial study was to assess the effect of community integrated management of childhood illness interventions on child health in Ile Ife, Osun State, Nigeria. Osun State has a population of about 3.4 million people (7). Ife Central Local Government was the study site while Ilesa East Local Government was the comparison site. Ife Central LGA implements CIMCI programme but Ilesa East LGA does not. There are 11 wards in each of the selected LGAs. The community in both LGAs comprise both rural and urban settlements arranged into streets.

Study design: The study was of a comparative cross-sectional design supplemented with a qualitative data collected through Focus Group Discussion (FGD).

Study population: The study population was caregivers of children of 0-59 months old, and their index children (the child selected for the study). Excluded from the study were caregivers who did not give consent or whose children were ill during the study period.

Sample size determination: The sample size was determined using a formula for comparing independent proportions (8). It was assumed, for calculating the sample size, that $10 \%$ difference

DOI: http://dx.doi.org/10.4314/ejhs.v28i2.13 
exists between implementing and nonimplementing LGAs (the assumption for this study was, care seeking prevalence is greater by $10 \%$ in the implementing LGA than nonimplementing LGA), a 5\% type I error and $80 \%$ power was also assumed (8). This gave a sample size of 722 caregivers and their index children aged 0-59 months old.

Sampling technique: A multistage cluster sampling technique was used. Three hundred and sixty-one respondents were selected from each of the LGA.

Stage 1: Ife Central LGA was purposively selected from the 30 LGAs in the state. The only LGA that implements CIMCI. Ilesa East LGA, the comparison LGA, does not implement CIMCI. The LGA was randomly selected from among the non-CIMCI-implementing LGAs in the state.

Stage 2: Wards 4 and 5 in Ife Central LGA implements CIMCI and were purposively selected. Random selection of Wards 2 and 8 among the 11 wards in Ilesa East LGA was done by balloting system.

Stage 3: The primary sampling units were the enumeration areas (EAs) of the two purposively selected (wards 4 and 5) and those of the two randomly selected wards (wards 2 and 8). Onefifth of the EAs in each LGA were selected using simple random selection. In Ife Central LGA 36 EAs were selected, while Ilesa East LGA, 30 EAs were selected. At each site, the sample size of 361 was divided by the number of the selected EAs to determine the number of respondents to be enrolled from each EA. An average of 10 respondents from selected EAs in Ife Central LGA and 12 respondents from selected EAs in Ilesa East LGA were recruited until the desired sample size of 361 was achieved in each LGA.

Stage 4: Household listings were done in the selected EAs in both LGAs. In each EA, one street was randomly selected by the ballot system. A household was randomly selected as the starting point in the selected street. Systematic random selection of households was done. Interviews began in the first eligible household until the targeted number of interviews were achieved.
Stage 5: The sampling unit was the household. Eligibility for participation in any visited household was the presence of a child 0-59 months. One caregiver with an eligible child was interviewed in every household that was selected. In situations where the household had more than one eligible subject, the child with a birthday nearest to the interview period was chosen.

Data collection procedures: A structured, pretested interviewer-administered questionnaire was used for data collection. The questionnaire was adapted from that previously used by UNICEF and validated by the WHO in Nigeria (9).

Data were collected on the sociodemographic and household characteristics, child healthcare practices and immunization history. Evidence of immunisation was obtained by sighting and assessing the immunisation card or by history. Vitamin A coverage rate coverage (defined as two doses within a 1-year period between ages 12 and 59 months) was sought by history and also by checking the immunisation card. A sample of the Vitamin A capsule commonly used was shown to the respondents to aid recall. Health education and mobilization session by CORPs in the implementing wards were randomly observed by principal investigator and research assistant to affirm and assess the process. Five appropriately trained research assistants were recruited to assist in data collection. For the qualitative part, six FGDs sessions were held. Two sessions with CORPs in the two implementing wards in Ife central LGA and four session with caregivers in the two LGA involved in the study.

Ethical clearance: Written informed consent was obtained from the study participants. Ethical clearance was obtained from the Ethics and Research Committee of Obafemi Awolowo University Teaching Hospitals Complex.

Data analysis: The data were analysed using SPSS version 16.0 (SPSS Inc., Chicago). Descriptive analysis was used for the sociodemographic characterization of the respondents. The chi-square $(\chi 2)$ test/likelihood ratio chisquare test was used to assess the statistical significance of the bivariate associations. Factors

DOI: http://dx.doi.org/10.4314/ejhs.v28i2.13 
that were significant at $(p<0.05)$ were then used in logistic regression models to estimate the independent effect of health education on child health practices and determinants.

\section{RESULTS}

As revealed in Table 1, 722 caregivers with children of 0-59 months old were involved in the study. Over $(90 \%)$ of the caregivers were married in both LGAs. More than half (57.9\% and 54\%) of the index children from the implementing LGA and non-implementing LGA were males. Their age distribution shows that the majority of the index children in the CIMCI implementing LGA (31.6\%) were aged between 24-35 months and in the non-implementing LGA most (21.9\%) were aged between 36-59 months. No statistically significant difference exists in wealth index between the two areas $(\mathrm{p}=0.344)$. More caregivers in the implementing LGA $(79.8 \%)$ could provide the child health card compared to $55.4 \%$ in the non-implementing LGA $(\mathrm{p}<0.001)$.

Table 1: socio-demographic characteristics of mothers and index children by LGA in Osun State, Nigeria (June 2013).

\begin{tabular}{|c|c|c|c|}
\hline Characteristics & $\begin{array}{l}\text { CIMCI implementing } \\
\text { LGA N=361 } \\
\text { n (\%) }\end{array}$ & $\begin{array}{l}\text { NON-CIMCI LGA } \\
\mathrm{N}=361 \\
\mathrm{n}(\%)\end{array}$ & $\begin{array}{l}\text { Statistical Indices } \\
\mathrm{p}\end{array}$ \\
\hline \multicolumn{4}{|l|}{ Index children age (months) } \\
\hline$\leq 5$ & $29(8.0)$ & $55(15.2)$ & \multirow{5}{*}{$0.001^{*}$} \\
\hline $6-11$ & $47(13.0)$ & $82(22.7)$ & \\
\hline $12-23$ & $61(16.9)$ & $75(20.8)$ & \\
\hline $24-35$ & $114(31.6)$ & $70(19.4)$ & \\
\hline $36-59$ & $110(30.5)$ & $79(21.9)$ & \\
\hline \multicolumn{4}{|l|}{ Index Children Sex } \\
\hline Male & 209(57.9) & $195(54.0)$ & \multirow{2}{*}{0.290} \\
\hline Female & $152(42.1)$ & $166(46.0)$ & \\
\hline \multicolumn{4}{|c|}{ Availability of child health card } \\
\hline Yes & $288(79.8)$ & $200(55.4)$ & \multirow[t]{2}{*}{0.001} \\
\hline No & $73(20.2)$ & $161(44.6)$ & \\
\hline \multicolumn{4}{|l|}{ Maternal age (years) } \\
\hline$\leq 30$ & $178(49.3)$ & $170(47.1)$ & \multirow[t]{2}{*}{0.552} \\
\hline$>31$ & $183(50.7)$ & 191(52.9) & \\
\hline \multicolumn{4}{|l|}{ Maternal Education } \\
\hline Below secondary education & $87(24.1)$ & $38(10.5)$ & \multirow[t]{2}{*}{0.001} \\
\hline Secondary and above & 274(75.9) & $323(89.5)$ & \\
\hline \multicolumn{4}{|l|}{ Marital Status } \\
\hline Married & 339(93.9) & $348(96.4)$ & \multirow{2}{*}{0.119} \\
\hline Not married & $22(6.1)$ & $13(3.6)$ & \\
\hline \multicolumn{4}{|l|}{ Maternal Occupation } \\
\hline Housewives & $18(5.0)$ & $39(10.8)$ & \multirow{2}{*}{0.004} \\
\hline Not Housewives & $343(95.0)$ & $322(89.2)$ & \\
\hline \multicolumn{4}{|l|}{ Wealth Index } \\
\hline First and Second quintile & $234(64.8)$ & $246(68.1)$ & \multirow{2}{*}{0.344} \\
\hline Third and above & $127(35.2)$ & $115(31.9)$ & \\
\hline
\end{tabular}

*Likelihood chi-square test

Amongst children that are 9 months old and above, vitamin A supplementation before the study was received by $89.1 \%$ and $79.8 \%$ of the children in implementing and non-implementing LGAs respectively (Table 2). Of those children aged 12-23 months, 93.4\% received DPT3 in

DOI: http://dx.doi.org/10.4314/ejhs.v28i2.13 
implementing area and $66.7 \%$ in nonimplementing area $(\mathrm{p}<0.001)$. Among index children aged $12-23$ months, $91.8 \%$ received measles vaccine in the implementing area compared to $64 \%$ in the non-implementing area. This difference was statistically significant $(\mathrm{p}<0.001)$.

Table 2: Immunization and Vitamin A supplementation status by LGA in Osun State, Nigeria (June 2013)

\begin{tabular}{|c|c|c|c|}
\hline Variable & $\begin{array}{l}\text { CIMCI } \\
\text { implementing } \\
\text { LGA } \\
\text { n(\%) }\end{array}$ & NON-CIMCI LGA & Statistical Indices \\
\hline $\begin{array}{l}\text { Children older than } 9 \\
\text { months Ever had Vitamin } A^{*}\end{array}$ & $\mathrm{~N}=312$ & $\mathrm{~N}=263$ & \\
\hline Yes & $278(89.1)$ & $210(79.8)$ & \multirow{3}{*}{$\mathrm{P}=0.002$} \\
\hline No & $34(10.9)$ & $53(20.2)$ & \\
\hline Total & $312(100.0)$ & $263(100.0)$ & \\
\hline $\begin{array}{l}\text { DPT3 Vaccine Received } \\
\text { Children Age 12-23 Month }\end{array}$ & $\mathrm{N}=61$ & $\mathrm{~N}=75$ & \multirow{4}{*}{$\mathrm{P}<0.001$} \\
\hline Yes & $57(93.4)$ & $50(66.7)$ & \\
\hline No & $4(6.6)$ & $25(33.3)$ & \\
\hline Total & $61(100.0)$ & $75(100.0)$ & \\
\hline $\begin{array}{l}\text { Measles Vaccine Received } \\
\text { Children Age 12-23 Month" }\end{array}$ & & & \\
\hline Yes & $56(91.8)$ & $48(64.0)$ & \multirow{3}{*}{$\mathrm{P}<0.001$} \\
\hline No & $5(8.2)$ & $27(36.0)$ & \\
\hline Total & $61(100.0)$ & $75(100.0$ & \\
\hline
\end{tabular}

Regarding hand washing, after toilet use, 91.1\% of caregivers in the implementing LGA and $79.8 \%$ in the non-implementing $\operatorname{LGA}(\mathrm{p}<0.001)$ wash their hands after using the toilets. About $60.1 \%$ of caregivers reported washing of hands before preparing meals in the implementing LGA compared with $45.1 \%$ in non-implementing LGA (Table 3). Over $75 \%$ wash their hands before feeding the child in the implementing LGA, but only $55.1 \%$ do in the non-implementing $(\mathrm{p}<0.001)$. The practice of using soap while washing hands was higher in implementing $(83.7 \%)$ than the non-implementing area $(66.8 \%)$. Fewer households in the non-implementing area $64.5 \%$ had a soap and water container in the toilet compared to $85.3 \%$ in the implementing area. This difference was statistically significant $(\mathrm{p}<0.001)$ (Table 3).

About $86.4 \%$ of households in the implementing area use pipe and protected well as source of drinking water while only $56.2 \%$ use a similar source in the non-implementing LGA (Table 4). Toilet facilities were available in over $92 \%$ of households in both LGAs. The commonest type of toilet in both LGA was Pit latrine $62.75 \%$ in the implementing LGA and $57.1 \%$ in the nonimplementing LGA $(p=0.128)$. More caregivers in the implementing LGA (59.3\%) dispose of children faeces by throwing in the toilet than in the non-implementing LGA (55.1\%) (Table 4). 
Table 3: Hand washing practices of mothers in CIMCI implementing and non-implementing LGA in Osun Nigeria (Jun (2013).

\begin{tabular}{llll}
\hline Hand washing practices & $\begin{array}{l}\text { CIMCI implementing } \\
(\mathbf{N}=\mathbf{3 6 1}) \\
\mathbf{n}(\mathbf{\%})\end{array}$ & $\begin{array}{l}\text { NON-CIMCI LGA } \\
\mathbf{( N = 3 6 1 )} \\
\mathbf{n}(\mathbf{\%})\end{array}$ & $\begin{array}{l}\text { Statistical } \\
\text { Indices }\end{array}$ \\
\hline Before feeding the child & $273(75.6)$ & $199(55.1)$ & $\mathrm{P}<0.001$ \\
Before preparing meal & $217(60.1)$ & $163(45.1)$ & $\mathrm{P}<0.001$ \\
Before eating & $263(72.9)$ & $234(64.8)$ & $\mathrm{P}=0.020$ \\
After using toilet & $329(91.1)$ & $288(79.8)$ & $\mathrm{P}<0.001$ \\
After child defecated & $298(82.5)$ & $261(72.3)$ & $\mathrm{P}=0.001$ \\
With soap and water & $302(83.7)$ & $241(66.8)$ & $\mathrm{P}<0.001$ \\
$\begin{array}{l}\text { Soap and water container in } \\
\text { the toilet }\end{array}$ & $308(85.3)$ & $233(64.5)$ & $\mathrm{P}<0.001$ \\
\hline
\end{tabular}

Table 4: Source of water, availability of toilets facilities and handling of index children faeces by LGA Osun State, Nigeria (June 2013).

\begin{tabular}{lccc}
\hline & $\begin{array}{c}\text { CIMCI implementing } \\
\text { LGA (N=361) } \\
\mathbf{n}(\mathbf{\%})\end{array}$ & $\begin{array}{c}\text { NON-CIMCI LGA } \\
\mathbf{( N = 3 6 1 )} \\
\mathbf{n}(\mathbf{\%})\end{array}$ & Statistical Indices \\
Variable & & & \\
\hline $\begin{array}{l}\text { Source of Drinking Water } \\
\text { Pipe \& Protected well }\end{array}$ & $312(86.4)$ & $203(56.2)$ & $\mathrm{P}<0.001$ \\
$\quad \begin{array}{l}\text { Unprotected well \& others } \\
\text { Availability of Toilet }\end{array}$ & $49(13.6)$ & $158(43.8)$ & \\
$\quad$ Yes & $333(92.2)$ & $335(92.8)$ & \\
$\quad$ No & $28(7.8)$ & $26(7.2)$ & $\mathrm{P}=0.777$ \\
Types of Toilets & & & \\
Pit latrine & $226(62.6)$ & $206(57.1)$ & \\
$\quad$ Water Closet & $107(29.6)$ & $129(35.7)$ & $\mathrm{P}=0.218$ \\
$\quad$ None & $28(7.8)$ & $26(7.2)$ & \\
Handling of index child & & & \\
Faeces & & & \\
$\quad$ Child uses toilets & $122(33.8)$ & $97(26.9)$ & $\mathrm{P}<0.001$ \\
$\quad$ Thrown into toilets & $214(59.3)$ & $199(55.1)$ & \\
$\quad$ Rinsed while washing & $7(1.9)$ & $34(9.4)$ & \\
$\quad$ Thrown in the yard & $18(5.0)$ & $31(8.6)$ & \\
\hline
\end{tabular}

A multivariate logistic regression analysis was performed to assess independent effect of health education on selected child health determinants (Table 5). The result showed that children in the CIMCI implementing LGA were more likely to receive DPT Vaccine (Adjusted odds ratio (AOR) 3.02, 95\% confidence interval (CI) 1.55-7.98), measles vaccine (AOR 5.56, 95\% CI 2.76-12.54). There was statistical difference in hand washing practices between the two LGA. Caregivers in the
CIMCI implementing LGA were four times more likely to wash hand with soap and water than in the non-implementing LGA (AOR 4.01, 95\% CI 2.43-5.98). Similarly, they were almost four times more likely to observe hand washing practice before feeding their child (AOR 3.70, $95 \%$ CI 1.58-7.72). Handling of index child faeces was about six and a half times more properly done in the Implementing LGA than none implementing LGA (AOR 6.50, 95\% CI

DOI: http://dx.doi.org/10.4314/ejhs.v28i2.13 
2.66-13.16). This association was found to be so strong even after confounding variables were controlled for. In all the cases after adjusting for confounding variable such as (maternal age, education level, wealth index, maternal occupation, marital status, child's age), the associations were still statistically significant (Table 5) except for the availability of toilets and types of toilets which were not significantly different even at the bivariate level.

Table 5: Effect of Health education on selected Child Health Determinant in Ile Ife, Osun State, Nigeria (2013)

\begin{tabular}{|c|c|c|c|c|}
\hline Variable & $\begin{array}{l}\text { CIMCI } \\
\text { implementing } \\
\text { LGA }\end{array}$ & $\begin{array}{l}\text { NON- } \\
\text { CIMCI } \\
\text { LGA }\end{array}$ & $\begin{array}{l}\text { Crude OR } \\
(95 \% \mathrm{CI})\end{array}$ & $\begin{array}{l}\text { Adjusted OR } \\
(95 \% \mathrm{CI})\end{array}$ \\
\hline \multicolumn{5}{|c|}{$\begin{array}{l}\text { Children older than months Ever } \\
\text { had Vitamin A* }\end{array}$} \\
\hline Yes & $278(89.1)$ & $210(79.8)$ & & \\
\hline No & $34(10.9)$ & $53(20.2)$ & $1.22(1.04-2.20)$ & $1.53(1.22-5.24)$ \\
\hline \multicolumn{5}{|l|}{$\begin{array}{l}\text { DPT3 Vaccine Received } \\
\text { Children Age 12-23 Month }\end{array}$} \\
\hline Yes & $57(93.4)$ & $50(66.7)$ & $2.01(1.02-2.68)$ & $3.02(1.55-7.98)$ \\
\hline No & $4(6.6)$ & $25(33.3)$ & & \\
\hline \multicolumn{5}{|l|}{$\begin{array}{l}\text { Measles Vaccine Received } \\
\text { Children Age 12-23 Month }\end{array}$} \\
\hline Yes & $56(91.8)$ & $48(64.0)$ & $3.51(2.40-9.89)$ & $5.56(2.76-12.54)$ \\
\hline No & $5(8.2)$ & $27(36.0)$ & & \\
\hline \multicolumn{5}{|l|}{ Source of Drinking Water } \\
\hline \multicolumn{5}{|l|}{ Pipe \& Protected well } \\
\hline \multicolumn{5}{|l|}{ Unprotected well \& others } \\
\hline Yes & $312(86.4)$ & $203(56.2)$ & $2.02(1.60-2.91)$ & $2.29(1.09-3.64)$ \\
\hline No & $49(13.6)$ & $158(43.8)$ & & \\
\hline \multicolumn{5}{|c|}{$\begin{array}{l}\text { Hand Washing before preparing } \\
\text { food }\end{array}$} \\
\hline Yes & $217(60.1)$ & $163(45.1)$ & $1.15(1.01-3.27)$ & $1.50(1.12-4.56)$ \\
\hline No & 144(39.9) & $198(54.9)$ & & \\
\hline \multicolumn{5}{|c|}{ Hand washing before feeding Child } \\
\hline Yes & $273(75.6)$ & $199(55.1)$ & & \\
\hline No & $88(24.4)$ & $162(44.9)$ & $3.35(1.68-6.98)$ & $3.70(1.58-7.72)$ \\
\hline \multicolumn{5}{|c|}{ Hand washing with soap and water } \\
\hline Yes & $302(83.7)$ & $241(66.8)$ & & \\
\hline No & $59(16.3)$ & $120(33.2)$ & $3.55(2.22-6.68)$ & $4.01(2.43-5.98)$ \\
\hline \multicolumn{5}{|l|}{$\begin{array}{l}\text { Hand washing after Child } \\
\text { defeacted }\end{array}$} \\
\hline Yes & $298(82.5)$ & $261(72.3)$ & $1.25(1.05-2.88)$ & $1.63(1.55-3.99)$ \\
\hline No & $68(17.5)$ & $100(27.7)$ & & \\
\hline \multicolumn{5}{|l|}{ Handling of index child } \\
\hline Disposed into toilet & $336(93.1)$ & 296(81.9) & $4.56(2.30-12.36)$ & $6.50(2.66-13.16)$ \\
\hline Disposed outside toilet & $25(6.9)$ & $65(18.01)$ & & \\
\hline
\end{tabular}

DOI: http://dx.doi.org/10.4314/ejhs.v28i2.13 


\section{DISCUSSION}

The study assessed the effect of health education activities on selected child health determinants such as immunisation, hand washing practices and sanitation in Ile Ife, Nigeria. The benefits of immunisation are numerous; immunising late is better than not immunising at all. Full and adequate immunisation can reduce child mortality remarkably. DTP3 coverage is used as a proxy indicator for the coverage of immunisation services and programme performance (10). This study assessed DPT3 and measles coverage for children aged 12-23 months in the CIMCI implementing and non-implementing LGAs. A large difference was noted in DPT coverage between the LGAs. Nine in ten of the children in the implementing LGA had received DPT3 compared to six in ten in the non-implementing LGA. The difference was statistically significant although the rate in both LGAs was higher than the national rate.

This achievement is quite remarkable and may not be unconnected with the influence of tertiary health facility and availability of multiple health facilities offering immunisation services in both LGAs. However, the influence of health education and effective mobilisation by CORPs may explain the difference between the two LGAs. FGD findings established that CORPs always mobilise caregivers to take children for immunisation on routine immunisation days or national immunisation days. This finding is in keeping with results from the Philippines where it was reported that social mobilisation increased coverage by $11 \%$ and by $12 \%$ in Cambodia $(11,12)$.

About nine-in-ten children in the implementing LGA received measles vaccines compared to six-in-ten in the non-implementing LGA. This difference was also statistically significant. This finding is supported by FGD report which suggested that the activities of the CORPs resulted in increased utilisation and access to immunisation schedules within the first year of life in the implementing LGA. Thus, using measles vaccine as a proxy, more children in the implementing LGA were more likely to complete immunisation than those in the non-implementing LGA. Health education, communication and mobilisation providing information about details of the time, place and age for routine vaccinations have been found to successfully increase coverage (11).

Vitamin A is essential for effective immunesystem functioning. It is established that even moderate deficiency increases a child's risk of mortality and severe infection (13). On comparing Vitamin A coverage between the two LGAs, the study found that Vitamin A coverage was high in both LGAs. However, the rate in the CIMCI implementing LGA was higher than the nonimplementing one with statistical difference between the two LGAs. The high rate of Vitamin A coverage of almost $90 \%$ in the implementing LGA is of note and may not be unrelated to the efforts of the CORPs as they educate mothers on health benefits of vitamin A. Another factor might also be the supplementation that takes place during polio eradication campaigns. A Similar high rate was found by Ebuchi in a study in the same area previously (14). However, the rate from this study was much higher than in the study. This may be explained by the continued effort at health education and communication by the CORPs in the CIMCI implementing LGA.

Poor hygiene practices are associated with diarrhoeal disease. In this study, nine-in-ten caregivers washed their hands after using the toilet in the implementing LGA while eight in ten does in the non-implementing LGA. This proportion is surprisingly high in both groups; this can be due to over-reporting and increased expression of expected behaviour by the caregivers. According to several studies, measuring handwashing behaviour is problematic, as good behaviours are likely to be increased under observation and over reported in surveys (15-17). The high rate found in this study may be due to these facts. A previous study in area (14) two years after implementation of CIMCI reported that less than half of caregivers wash their hands with soap and water unlike the high rate of $83.7 \%$ observed in this study. This

DOI: http://dx.doi.org/10.4314/ejhs.v28i2.13 
observation is most likely due to the sustained health education effort as a result of CIMCI implementation. This finding is supported by Halder et al who reported that soap use for handwashing was more common for each of critical times except serving food (16).

Source of drinking water was statistically different; however, availability of toilets and types of toilets were not significantly different in the two LGAs. The significant difference observed in water source may not be unconnected with the activities of some NGOs in the implementing LGA in the provision of clean water, unlike toilet facilities. In this study, $5 \%$ of houses had human faeces around the yards, dumps or houses in the implementing area lower than the prevalence of the $8.6 \%$ in the non-implementing LGA. The lower rate observed in this study in the implementing LGA may be ascribed to the effect of CIMCI considering that higher values have been reported in other parts of Nigeria even in more recent studies (18). There is, therefore, a need for health education aimed at sustaining the practice of caregivers regarding disposal of faeces and also to sustain the gains in handwashing practices. The strength of this study is that it has revealed the effect of sustained community level health education effort on child health practices and determinants. One limitation of the study is in the fact that caregivers may not reveal their actual practice for fear of unknown action. This was, however, minimised by interviewers first gaining the confidence of the respondents. There was also no measure of the effect of exclusion of caregivers with sick children during the study. Another issue is the cross-sectional study design, which measured the exposure and outcome at the same time, and cannot measure cause and effect relationships.

In conclusion, our findings suggest that child health determinant in communities can be improved with appropriate health education activities and health-promoting knowledge and practices. Health education and mobilisation activities should be scaled up to improve coverage of appropriate community practices.

\section{REFERENCES}

1. Federal Ministry of Health. Newborn Health in the context of the Integrated Maternal, Newborn and Child Health Strategy Executive Summary Revised 2nd edition, 2011.

2. Beaton $\mathrm{GH}$, Martorell $\mathrm{R}$, Aronson $\mathrm{KJ}$, Edmonston B, McCabe B, Ross AC etal. 'Effectiveness of Vitamin A Supplementation in the Control of Young Child Morbidity and Mortality in Developing Countries', ACC/SCN State-of-the-Art Series, Nutrition. 1993 Policy Paper No. 13, Geneva.

3. World Health Organisation. WHO and Maternal Child Epidemiology Estimation (MCEE) cause of death estimates 2015. Availablefromhttp://www.who.int/healthinfo/ global_burden_disease/estimates_childCOD methods_2000_2015/en.Accessed 23/1/2017.

4. World Health Organisation/United Nations Children's Fund. The role of IMCI in improving family and Community practices to support health and development. Available fromhttp://www.whoint/child-adolesenthealth 1999. Accessed 20/11/2011.

5. World health Organisation/Child health Development. Improving family and community practices; A component of the IMCI strategy. Bull of WHO. 1998; 73(15) : 119-128

6. Ogundele O.A, Ogundele T, Olajide O.S, Agunbiade O. I. Effect of community integrated management of childhood illness on mothers' healthcare-seeking behaviour and home management of childhood illness in IleIfe, South-West Nigeria: A household survey. $S$ Afr J Child Health 2016; 10(1):16-19. DOI: 10.7196/SAJCH. 2016.v106i1.912.

7. Ministry of Health Osogbo. Osun State Health Facilities Inventory. Osogbo, Nigeria: Ministry of Health Osogbo, 2009:7-8.

8. Lwanga S K C. World Health Organisation. Planning a health survey, teaching health statistics Yvok Teds 1986:149-60.

9. United Nations Children's Fund/Federal Ministry of health. IMCI in the Hands of Families: Nigeria Country Report of Baseline

DOI: http://dx.doi.org/10.4314/ejhs.v28i2.13 
Studies on Key Family and Community Practices in IMCI-implemented LGAs. Abuja, Nigeria. Federal Ministry of Health. 2005.

10. GAVI alliance. Guidelines on country proposals-for support for: new and underused vaccines. Geneva, GAVI Alliance, 2011.

11. World Health Organisation/United Nation children's Fund. Increasing immunization coverage at health facility level. Available from.http://www.who.int/iris/bitstream/10665 /67791/1/WHO_V\%26B_02.27.pdf Accessed 22/11/2011

12. Zelee H, Betty K, Kareen E. Family and community practices that promote child survival, growth and development : a review of the evidence. Available from http://www.who.int/iris/bitstream/pdf. Accessed 20/11/2011

13. World Health Organization, The World Health Report 2002: Reducing risks, promoting healthy life, WHO, Geneva, 2002, p. 55 .

14. Ebuehi OM. Health care for under-fives in Ile-Ife, South-West Nigeria: Effect of the Integrated Management of Childhood Illnesses (IMCI) strategy on growth and development of under-fives. Afr J Prm Health Care Fam Med; 2009; 1(1), Art. \#29, 8 pages. DOI:10.4102/phcfm.v1i1.29.

15. Halder, AK, Molyneaux, JW, Luby S P, Ram PK Impact of duration of structured observations on measurement of hand washing behavior at critical times. $B M C$ Public Health 2013;13:705.

16. Ram PK, Halder AK, Granger SP, Jones T, Hall P, Hitchcock D et al: Is structured observation a valid technique to measure handwashing behavior? Use of acceleration sensors embedded in soap to assess reactivity to structured observation. Am J Trop Med Hyg, 2010; 83:1070-1076 10.4269.

17. Manunebo M, Haggerty PA, Muladi K, Kirkwood BR. Measuring hygiene practices: a comparison of questionnaires with direct observations in rural Zaïre. Trop Med \& Intl Hlth, 1997; 2(11):1015-1021.

18. Aluko OO, Afolabi OT, Olaoye EA, Adebayo $\mathrm{AD}$, Oyetola SO, Abegunde OO. The management of the faeces passed by under five children: an exploratory, cross-sectional research in an urban community in Southwest Nigeria. BMC Public Health. 2017; 17:178. doi:10.1186/s12889-017-4078-1. 